\title{
Systematic Analysis of the Offset-PLL Output Spur Spectrum
}

\author{
Ching-Feng Lee and Song Tsuen Peng, Fellow, IEEE
}

\begin{abstract}
This paper presents a mechanism that explains exactly the cause of spurs at the output of offset-PLL (OPLL) transmitters. By a rigorous analysis of the relationship between the signals and disturbances in OPLL, accurate formulas are obtained which elucidate systematically the intermodulation nature of the output spur spectrum. From the analysis, it is shown that in practical situations the OPLL spurs are due to the disturbances from the voltage-controlled oscillator leakage, the local oscillator leakage, and the image signal, rather than other spurious products of the offset mixer in the loop as previously explained. The formulas give insight into OPLL circuit design and frequencies accommodation and clearly depict the output spur distribution in the more realistic situation when the input is modulated. Finally, the formulas are verified by some elaborate experiments with extensive numerical results.
\end{abstract}

Index Terms - Intermodulation, leakage, local oscillator (LO), offset mixer, offset-PLL (OPLL), spurs, voltage-controlled oscillator (VCO).

\section{INTRODUCTION}

$\mathbf{F}$ OR THE global system for mobile communications (GSM) applications, a mixer in the loop, as illustrated in Fig. 1, is widely used. The main advantage of an offset-PLL (OPLL) is that it up-converts the modulated IF signal to the RF carrier frequency and meanwhile shapes out the broad-band phase noise of $I / Q$ modulator by the inherent loop filtering [1]-[5]. Usually, when a constant-frequency signal is applied to the input of OPLL, spurious products also appear along with the desired fundamental at the voltage-controlled oscillator ( $\mathrm{VCO}$ ) output. Although the phenomenon had been observed in previous papers [1]-[4], no reasons were interpreted clearly. In this paper, we propose a new mechanism that explains exactly the cause of spurs in the realistic IC operating conditions. By the rigorous analysis of the relationship between the signals and disturbances in OPLL, the phenomenon of spurs can be accurately predicted in a systematic way. Formulas derived from the proposed mechanism provide insight into circuit design and frequency planning in OPLL. Besides, the spur distribution in the case of input modulation can also be evaluated intuitively from the viewpoint of the proposed mechanism.

This paper is organized as follows. Section II first reviews the previous works related to the spur spectrum and then explains the cause of spurs in the realistic situation. A term called

Manuscript received December 13, 2004; revised March 19, 2005. This work was supported in part by the Industrial Technology Research Institute, Taiwan, R.O.C.

The authors are with the Department of Communication Engineering, National Chiao Tung University, Hsin-Chu 310, Taiwan, R.O.C. (e-mail: lcf@itri.org.tw).

Digital Object Identifier 10.1109/TMTT.2005.854221

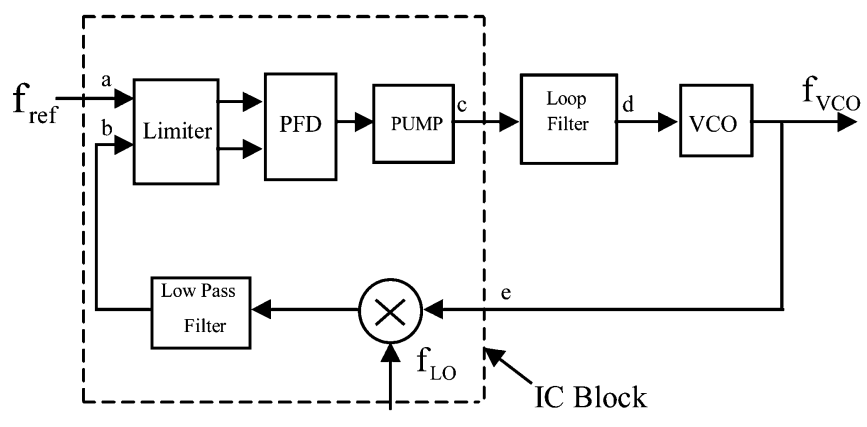

Fig. 1. Block diagram of the OPLL.

residual frequency is shown to be the consequence of the relationship between the reference IF and the feedback disturbance in a locked PLL.

In Section III, the predicting formula for the output spur spectrum is rigorously derived. The pulsewidth equation of the disturbance is solved and the concept of sampling theorem is applied to deal with the spectrum problem. Various nonideal situations such as the dead zone and mismatches in the circuits are discussed. The spur spectrum in the situation with some disturbances coexisting is also captured by solving the general pulsewidth equation. Formulas to determine suitable IF frequencies for spur suppression are then proposed.

In Section IV, the spur distribution in the case of input modulation in GSM is conceptually demonstrated and the strength reduction in spurs due to the modulation is explained. Both experimental and numerical results are given in Section V to verify the derived formulas. Also, the strength of the disturbance that cannot be probed from outside is estimated by the formulas. Finally, the conclusion is given in Section VI.

\section{REVIEW OF PREVIOUS WORKS AND FundAMENTALS OF THE CAUSE OF SPURS}

In the fairly few papers that talked about OPLL output spurs, the phenomenon was investigated by computer simulation only. In [2], the phase frequency detector (PFD) in the loop is treated as an ideal multiplier and the harmonic balance simulation is applied to convolute the IF, VCO, and local oscillator (LO) frequencies for all of the possible spurs. In [3], the open-loop dc conversion gain of the PFD loop filter is simulated at various IF harmonics, and the gain plot is interpolated to depict spurs. Since in both approaches the behavior of the PFD is not thoroughly understood, neither the cause of spurs were systematically explained, nor could any idea be given to predict their distribution in the realistic application when the input is modulated. 


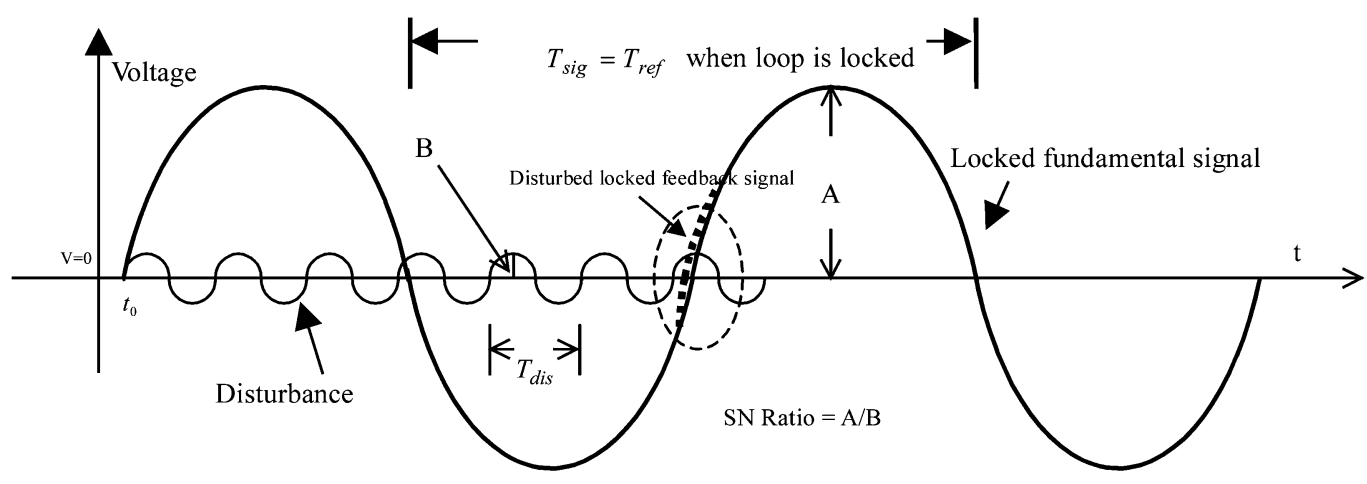

Fig. 2. Phase deviation caused by a small disturbance.

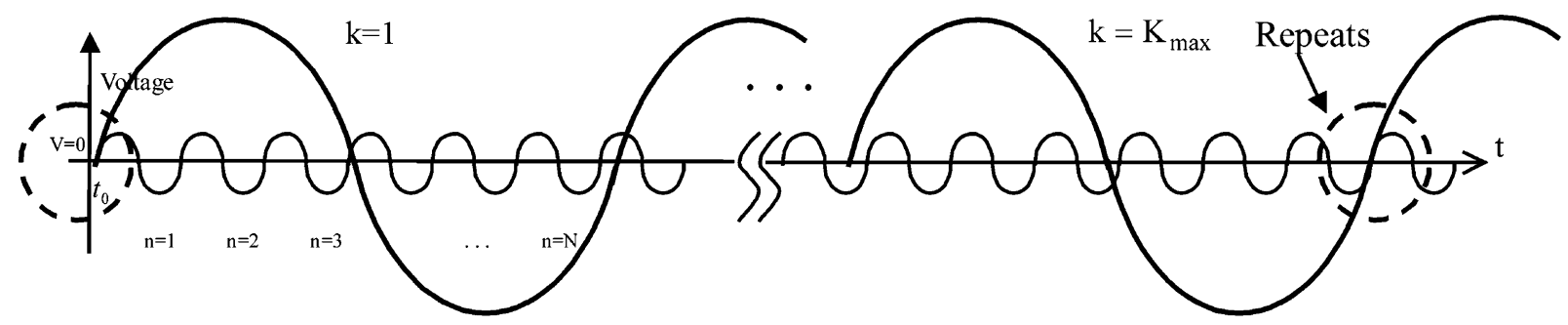

Fig. 3. Demonstration of the periodicity of phase-offset function.

For the better understanding of the cause of spurs, the operation principle of OPLL is described as below. As designated in Fig. 1, when OPLL is locked to a sinusoidal input at "a" in its steady state, the zero-crossing points of the feedback signal at "b" are coincident with those of the input. VCO is now controlled by a dc voltage and presents an RF sinusoidal output. In real situations, however, some disturbances coexist with the locked down-converted fundamental; therefore, the zero-crossing points of the fundamental signal are perturbed and deviate from those of the input reference, as shown in Fig. 2, where we assume that only one disturbance exists in the loop. This deviation series is sensed by PFD, and a train of current pulses is generated to feed the loop filter. As will be shown later, the deviation series presents periodicity and possesses specific frequency components. The VCO is thus modulated as in FM application and generates spurs at the output.

The periodicity of the zero-crossing deviations that are sensed at the rate of reference frequency can be checked using the frequency relation between the reference signal and feedback disturbances. For the case of a single disturbance, it is written as

$$
f_{\text {dis }}=N f_{\text {ref }}+f_{r}
$$

where $N$ is a positive integer and $0 \leq f_{r}<f_{\text {ref }}$. The $f_{r}$ is called the residual frequency. To view the relation in terms of phase offsets between them, (1) is rearranged as

$$
f_{\text {ref }}\left(\frac{1}{f_{\text {ref }}}-N \frac{1}{f_{\text {dis }}}\right)=f_{r} \frac{1}{f_{\text {dis }}}
$$

where $1 / f_{\text {ref }}-N / f_{\text {dis }}=T_{\text {ref }}-N T_{\text {dis }}$ is the total phase offset in terms of time in one reference-signal cycle.

The ratio $f_{\text {ref }} / f_{r}$ can be reduced to $p / q$ where $p$ and $q$ are coprime. Obviously, the period of this phase-offset function is $p T_{\text {ref. }}$. This is indicated in Fig. 3, where $p$ is set to $K_{\max }$ and the zero-crossing points of the reference signal and disturbance are assumed to coincide at $t=t_{o}$. Apparently $K_{\max }$ is the required minimum number of reference-signal cycles to overlap their zero-crossing points again.

It is noted that (2) can be interpreted as that, in one unit time interval where $f_{\text {ref }}$ cycles of reference signal have passed, there are exactly $f_{r}$ excessive cycles of disturbance elapsed. This implies that the phase-offset function experiences a total $2 \pi f_{r}$ radian of variation in this unit time interval and thus elucidates that the phase-offset function, i.e., the zero-crossing deviation series, is indeed a periodic function of time that contains a frequency component $f_{r}$.

In Section III, the spectrum of the generated current pulses is strictly analyzed. As will be found, it contains not only the frequency component $f_{r}$ but also its various harmonics at $m f_{r}$. Besides, due to the equivalent sampling effect inherent in the loop, it also contains components at $m f_{r} \pm n f_{\text {ref }}$.

\section{SPECTRUm ANALYSIS OF SPURS}

\section{A. Pulsewidth Equation}

In OPLL, input reference and feedback signals are in general fed to a high gain limiter before their differences in phase are extracted by PFD. Thus, current pulses caused by the zerocrossing deviations can be assumed to be ideal pulses with constant amplitude. A general aspect of these pulses for the case that $f_{\text {ref }}$ is an integer multiple of the residual frequency is depicted in Fig. 4, where the width of each pulse corresponds to the phase error between the perturbed feedback signal and the input reference.

The width and sign of each pulse can be determined from the following two classified relations. Fig. 5(a) describes a condition when $K T_{\text {dis }}<k\left(T_{\text {ref }}-N T_{\text {dis }}\right) \leq(K+0.5) T_{\text {dis }}$, where $K$ is an integer. In this condition, the disturbed signal leads the 


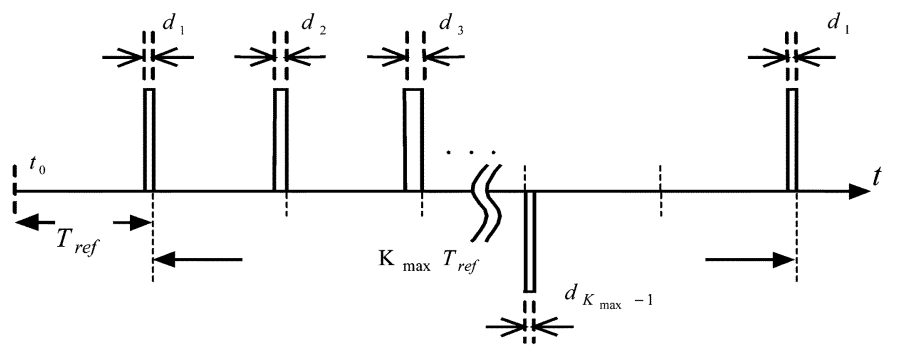

Fig. 4. Ideal current pulses caused by the disturbance.

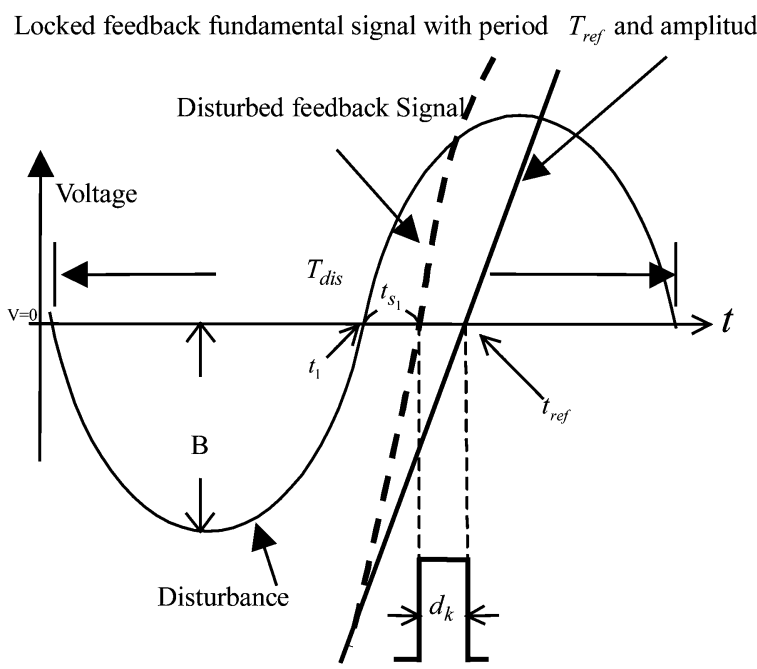

(a)

Locked feedback fundamental signal with period $T_{\text {ref }}$ and amplitude A

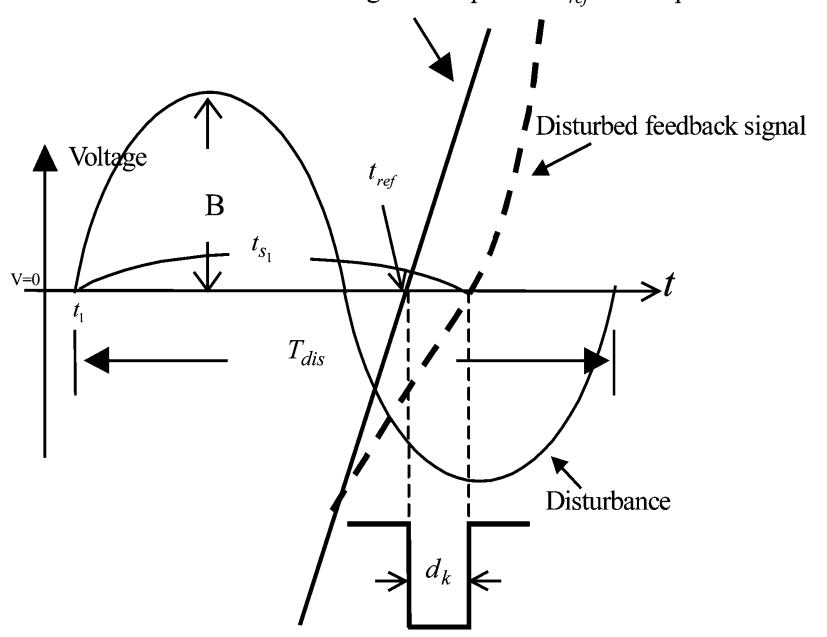

(b)

Fig. 5. Detailed phase relation between the reference and disturbed feedback signals. (a) The lead condition. (b) The lag condition.

reference and generates a positive pulse. The lag condition that produces a negative pulse is shown in Fig. 5(b), where $(K+$ $0.5) T_{\text {dis }} \leq k\left(T_{\text {ref }}-N T_{\text {dis }}\right)<(K+1) T_{\text {dis }}$. Note that in realistic situations the fundamental signal is much larger than the disturbance, so in each cycle the rising edge of the disturbed signal crosses the zero only once, as depicted in Fig. 5.
According to Fig. 5, zero-crossing points of the disturbed feedback signal satisfy the equation

$$
A \sin \left(2 \pi \frac{d_{k}}{T_{\text {ref }}}\right)-B \sin \left(2 \pi \frac{t_{s_{1}}}{T_{\text {dis }}}\right)=0
$$

where $A$ is the amplitude of the feedback fundamental signal, $B$ is the amplitude of the disturbance, and

$$
t_{s_{1}}=t_{\mathrm{ref}}-t_{1}-d_{k}=k T_{\mathrm{ref}} f_{r} T_{\mathrm{dis}}+t_{\phi}-d_{k} .
$$

In (4), $t_{\text {ref }}$ is the beginning of feedback fundamental in every cycle, $t_{1}$ is the beginning of a disturbance cycle preceding and nearest to $t_{\mathrm{ref}}, t_{s_{1}}$ is the difference between $t_{1}$ and the zerocrossing of perturbed feedback signal at its rising-edge, and $d_{k}$ is the corresponding zero-crossing deviation around the beginning of the $(k+1)$ th reference-signal cycle, where $k \in$ $\left\{0,1,2, \ldots, \mathrm{K}_{\max }-1\right\}$. Besides, $t_{\phi}$ is the initial time offset between the disturbance and the fundamental feedback signal.

Notice that, in Fig. 5(a), $d_{k}>0$ and, in Fig. 5(b), $d_{k}<0$. Using the fact that $\left|d_{k}\right|<<T_{\text {ref }}$ and the relation in (4), (3) can be rearranged as

$$
\sin \left(2 \pi f_{r} t+\phi_{\text {dis }}-2 \pi f_{\text {dis }} d_{k}\right)-2 \pi \frac{A}{B} f_{\text {ref }} d_{k}=0
$$

where $t=k T_{\text {ref }}, k \in\left\{0,1,2, \ldots, \mathrm{K}_{\max }-1\right\}$, and $\phi_{\text {dis }}=$ $2 \pi f_{\text {dis }} t_{\phi}$.

Equation (5) is the pulsewidth equation. It is solved in Appendix A by the method of power series approximation. The solution $d_{k}$, i.e., the pulsewidth function, is approximated as

$$
d_{k} \cong \frac{1}{2 \pi f_{\text {ref }}} \frac{B}{A} \sum_{m=1}^{\infty} g_{m}\left(\frac{B f_{\mathrm{dis}}}{A f_{\mathrm{ref}}}\right) \sin \left(m\left(2 \pi f_{r} t+\phi_{\mathrm{dis}}\right)\right)
$$

where $t=k T_{\text {ref }}$ and

$$
\begin{aligned}
& g_{1}(x)=1-\frac{1}{8} x^{2}+\frac{3}{32} x^{4}+\frac{45}{128} x^{6}+\frac{245}{256} x^{8}+\cdots \\
& g_{2}(x)=x\left(\frac{-1}{2}+\frac{1}{8} x^{2}-\frac{245}{256} x^{6}+\cdots\right) \\
& g_{3}(x)=x^{2}\left(\frac{3}{8}-\frac{5}{32} x^{2}+\frac{315}{256} x^{6}+\cdots\right) .
\end{aligned}
$$

$g_{m}(x)$ for $m \geq 4$ is negligible for small $x$.

Equation (6) declares that widths of pulses determined at discrete time instants follow a time function with components at the residual frequency and its harmonics.

\section{B. Spectrum of Spurs}

The spectrum of this periodic pulse series can be analyzed by the concept of the sampling theorem as explained below. First review that the Fourier transform of a narrow pulse $f_{p}(t)=1$ for a short duration $-d / 2 \leq t \leq d / 2$ is

$$
P_{s}(\omega)=\frac{2}{\omega} \sin \left(\frac{\omega d}{2}\right) .
$$




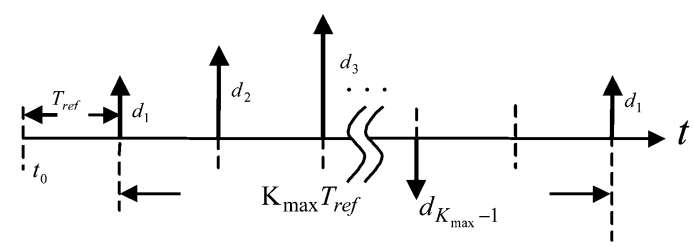

Fig. 6. Impulse sequence that approximates the pulse series in Fig. 4.

Thus, in the frequency range $f<<1 / 2 \pi d$, its spectrum almost behaves like an impulse of strength $d$. This indicates that, in the low-frequency band in which we are interested, the spectrum of the pulse sequence illustrated in Fig. 4 is almost equal to the spectrum of an impulse series with the corresponding strengths as shown in Fig. 6. In other words, in its low-frequency band, the spectrum of the original pulse sequence almost equals the spectrum of the pulsewidth function that is obtained by sampling the continuous function on the right-hand side of (6) at a rate of $f_{\text {ref. }}$. As derived in Appendix B, in the low-frequency band in which we are interested, the original periodic pulse sequence can be approximated as

$$
\begin{aligned}
Q_{\text {low }}(t) \cong \frac{I_{\mathrm{cp}} B}{2 \pi A} \sum_{n=-L_{2}}^{L_{2}} & \sum_{m=1}^{L_{1}} g_{m}\left(\frac{B}{A} \frac{f_{\text {dis }}}{f_{\text {ref }}}\right) \sin \\
& \times\left(2 \pi\left(m f_{r}+n f_{\text {ref }}\right) t+m \phi_{\text {dis }}\right)
\end{aligned}
$$

where $m=1,2,3, \ldots, L_{1}, n=0,1,2, \ldots, L_{2}, I_{\text {cp }}$ is the current pulse amplitude, and $L_{1}$ and $L_{2}$ are large positive integers.

Equation (8) clearly shows that the pulse sequence contains frequency components at $\pm\left(m f_{r} \pm n f_{\text {ref }}\right)$ in its low-frequency band. Note that, in general, these disturbing components are not very large and those with frequencies beyond the bandwidth of the loop filter are to be further attenuated. This implies that the output carrier of the VCO is modulated by a lot of signals with tiny modulation indexes [7] so that the generated spurs are only visible at the following frequency offsets from the output carrier:

$$
\begin{aligned}
f_{\text {spur }} & = \pm\left(m f_{r} \pm n f_{\text {ref }}\right) \\
& = \pm\left(m f_{\text {dis }}-(m N \pm n) f_{\text {ref }}\right)
\end{aligned}
$$

where $m=1,2,3 \ldots, L_{1}$ and $n=0,1,2, \ldots, L_{2}$.

Equation (9) systematically predicts the frequency positions of spurs. Note that the result is similar to the common intermodulation phenomenon usually encountered in nonlinear circuits. However, in OPLL, it is stemming from a totally new mechanism.

Note also that, among all of the miscellaneous spurs presented in (9), only the components with coefficient $g_{1}$ are noticeable. This is evident since in real cases the signal-to-noise ratio (SNR) $A / B$ is quite large, so the components in (8) with coefficients other than $g_{1}$ are very weak and only generate tiny spurs with strengths below the noise floor of VCO. Thus, generally speaking, at the output of OPLL, only the spurs at $\pm f_{r}$ and $\pm\left(f_{r}-n f_{\text {ref }}\right)$ offsets are observed.

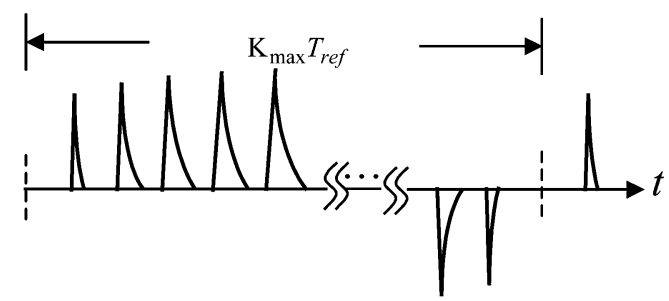

Fig. 7. Pulse sequence due to the finite gain of the charge pump.

It is noted that, for the spur located out of the loop bandwidth, the strength ratio $R_{\mathrm{sf}}$ of this spur to the VCO output carrier is

$$
\begin{aligned}
R_{\mathrm{sf}} & =\frac{J_{1}(\beta)}{J_{0}(\beta)} \cong \frac{\beta}{2} \\
& =\frac{B}{A} \frac{I_{\mathrm{cp}}}{4 \pi} g_{1}\left(\frac{B}{A} \frac{f_{\text {dis }}}{f_{\mathrm{ref}}}\right) G_{\mathrm{lf}} \frac{K_{\mathrm{vco}}}{2 \pi f}
\end{aligned}
$$

where $J_{i}$ is the Bessel function of order $i$ [9], $\beta$ is the equivalent modulation index of the corresponding component at $f$ in the pulse sequence, $G_{\text {lf }}$ is the response of the loop filter at $f$, and $K_{\mathrm{vco}}$ is the gain of the VCO. For spurs located inside the loop bandwidth, the feedback effect must be considered. As analyzed in Appendix D, considering the closed-loop effect, the strength ratio can be approximated as

$$
R_{\mathrm{sf}} \cong \frac{B}{A} \frac{I_{\mathrm{cp}}}{2 \pi} g_{1}\left|G_{\mathrm{lf}}\right| \frac{K_{\mathrm{vco}}}{4 \pi f} /\left|1+\frac{I_{\mathrm{cp}}}{2 \pi} g_{1} G_{\mathrm{lf}} \frac{K_{\mathrm{vco}}}{j 2 \pi f}\right| .
$$

In practical OPLL circuits, a charge pump never generates ideal current pulses due to the finite gain of transistors. As illustrated in Fig. 7, the amplitudes and shapes are nonlinearly dependent on the zero-crossing deviations and the asymmetry in each pulse is due to the unequal turning-on and -off times of the current sources in the pump. Therefore, the spectrum derived above must undergo a further strength adjustment and slight amplitude modulation to reflect this realistic condition. Besides, the inevitable dead zone of the PFD will decrease the width of the pulse to some extent or even swallow it. However, the dead zone also causes the feedback fundamental to drift randomly around the ideal locked state [8], so the effect of dead zone on the pulse sequence must be evaluated from an average point of view. Apparently, on average, the width of each pulse is to be reduced due to this effect. Two other situations are the input offset of the limiter and the mismatch between the charge and sink currents in the charge pump, both of which are resulting from the process, layout, and design mismatches. These effects will cause amplitude unbalance between the positive and negative pulses and generate additional spurs at $\pm f_{\text {ref }}$ offsets from the output carrier.

\section{Spurs in the Case of Multiple Disturbances in the Loop}

All of the derivations above consider a single disturbance only. In realistic situations, some disturbances may coexist in OPLL such as the VCO leakage, the LO leakage, the undesired image at $f_{\mathrm{LO}}+f_{\mathrm{VCO}}$, and other intermodulation terms due to 
the nonlinearity of the offset mixer in the loop. The spur distribution in this complex situation can be described by the general pulsewidth equation

$$
\sum_{i} B_{i} \sin \left(2 \pi f_{r_{i}} t+\phi_{d_{i}}-2 \pi f_{d_{i}} d_{k}\right)-2 \pi A f_{\mathrm{ref}} d_{k}=0
$$

where $B_{i}$ and $\phi_{d_{i}}$ are the amplitude and phase offset of the disturbance $i$ at frequency $f_{d_{i}}, A$ and $f_{\text {ref }}$ are the amplitude and frequency of the feedback fundamental signal, $f_{r_{i}}$ is the residual frequency of $f_{d_{i}}$ in reference to $f_{\text {ref }}$, and $t$ specifies the time in an interval designated by the index $k$ as detailed in Appendix C.

As derived in Appendix $\mathrm{C}$, the general pulsewidth function $d_{k}$ almost equals the superposition of respective pulsewidth functions of the presenting disturbances. Obviously, the noticeable spurs in this complex situation will be located at $\pm f_{r_{i}}$ and $\pm\left(f_{r_{i}}-n f_{\text {ref }}\right)$ offsets. However, as for the disturbances in practical OPLL circuits, the VCO leakage, the LO leakage, and the image at $f_{\mathrm{LO}}+f_{\mathrm{VCO}}$ are usually much larger than the RF-band intermodulation products of the offset mixer. On the other hand, those intermodulation products of the mixer such as $2 f_{\mathrm{LO}}-2 f_{\mathrm{VCO}}$ and $3 f_{\mathrm{LO}}-3 f_{\mathrm{VCO}}$, which fall into the low frequency band and receive not so much attenuation by the following low-pass filter, are apparently high-order terms with slight strengths only. Besides, the residual frequencies of these terms are all zero, i.e., they always keep constant phase differences with the reference input. Therefore, these disturbances only generate spurs at $\pm n f_{\text {ref }}$ offsets that are obviously far away from the signal band.

\section{Methods to Suppress Spurs}

1) Frequency Planning: Let $f_{r_{\mathrm{vco}}}$ denote the residual frequency of $f_{\mathrm{VCO}}$ in reference to $f_{\text {ref }}$. Then it is now clear that, in practical OPLL circuits, the appreciable spurs will occur at $\pm f_{r_{\mathrm{vco}}}, \pm\left(f_{r_{\mathrm{vco}}}-n f_{\mathrm{ref}}\right), \pm 2 f_{r_{\mathrm{vco}}}$, and $\pm\left(2 f_{r_{\mathrm{vco}}}-n f_{\mathrm{ref}}\right)$ offsets from the output carrier since $f_{\mathrm{LO}}$ shares the same residual frequency $f_{r_{\mathrm{vco}}}$ with $f_{\mathrm{VCO}}$ and the residual frequency of the image at $f_{\mathrm{LO}}+f_{\mathrm{VCO}}$ is $2 f_{r_{\mathrm{vco}}}$. Thus, for spurs not to appear within the interested low-frequency band bounded by $\pm \Delta f$ offsets in which $\Delta f<<f_{\text {ref }}$, the $f_{r_{\mathrm{vco}}}$ should satisfy

$$
\frac{f_{\mathrm{ref}}+\Delta f}{2}<f_{r_{\mathrm{vco}}}<f_{\mathrm{ref}}-\Delta f
$$

Equation (13) can be rearranged as

$$
\left(N+\frac{1}{2}\right) f_{\mathrm{ref}}+\frac{\Delta f}{2}<f_{\mathrm{VCO}}<(N+1) f_{\mathrm{ref}}-\Delta f .
$$

Equation (14) can be used to figure out suitable reference frequencies for OPLL so that no spurs could appear in the region close to the carrier. For the GSM band from 880 to $915 \mathrm{MHz}$, if no spurs are allowed within the range bounded by $\pm 4-\mathrm{MHz}$ offsets from the output carrier, then from (14) the suitable $f_{\text {ref }}$ is located in the range from 83.5455 to $83.619 \mathrm{MHz}$. If the forbidden region of spurs is bounded by $\pm 10-\mathrm{MHz}$ offsets, $f_{\text {ref }}$ should fall into the range from 102.78 to $102.94 \mathrm{MHz}$. When spurs only appear at positions far away from the output carrier,

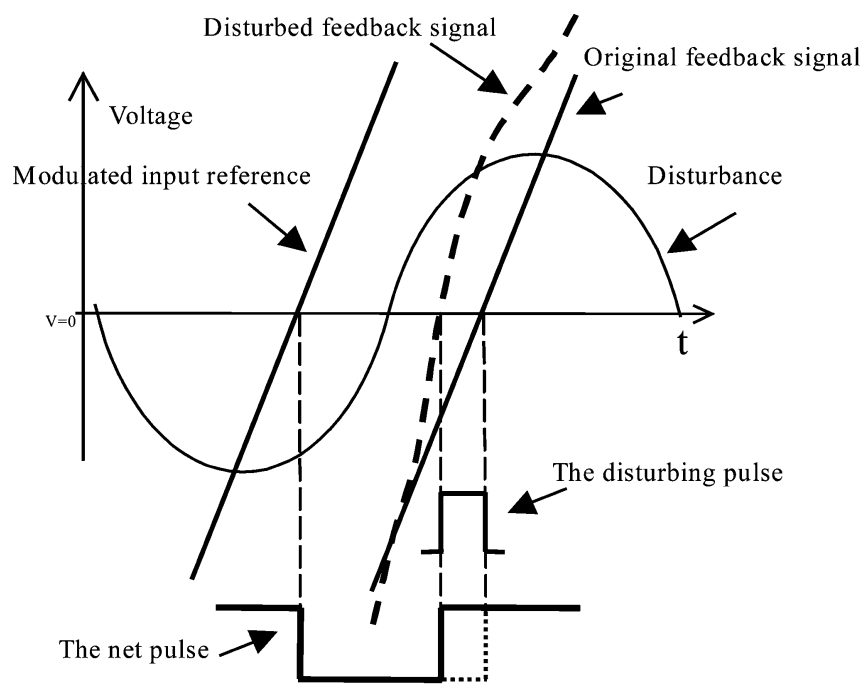

Fig. 8. Phase relation of the modulated input and the disturbed feedback in the phase-tracking process and the change of the actuated pulse in width.

their strengths are much more reduced because of the greater attenuation provided by the loop filter and the smaller modulation index of the disturbing component.

2) Circuit Design and Layout Considerations: To get rid of spurs thoroughly, the notorious disturbances such as the leaked LO and VCO signals from the mixer and the image at $f_{\mathrm{LO}}+$ $f_{\mathrm{VCO}}$ must be suppressed effectively. This requires reducing the layout and signal mismatches in the offset mixer [7] and the extensive attenuation at the RF band in the low-pass filter. Note that, in real situations, the external VCO usually provides a single output only, thus to reduce the mismatch the VCO signal should be converted to well-balanced ones before being fed to the balanced-type mixer. Reducing the gain of the limiter also suppresses spurs to some extent, but at the cost of desensitizing the phase-tracking capability of the OPLL. Decreasing the amplitude of the current sources in the charge pump reduces directly the strength of the spurs. However, this will decrease the loop bandwidth, i.e., the output frequency of the $\mathrm{VCO}$ will take a longer time to settle when OPLL changes the LO frequency to select another transmission channel.

\section{OUtPUt SPUR SPeCtrum FOR THE InPUT MODULATION CASE IN GSM}

For the realistic application when the input is modulated, the spur distribution can also be evaluated by the proposed mechanism. Suppose the loop is under control. Then the downconverted feedback signal tracks the modulated input quite well except that some phase difference exists so that the PFD is actuated to up-convert the phase information in the input to VCO output. If in this phase-tracking process there is also a disturbance presenting, the duration of the original actuated pulse will change a little, as shown in Fig. 8. Obviously the net pulse is the superposition of the original actuated pulse and the perturbing pulse caused by the disturbance. Thus, the spur distribution can be figured out by calculating the spectrum of this perturbing pulse sequence alone. Note that, for GSM applications, the data rate is much lower than the intermediate 


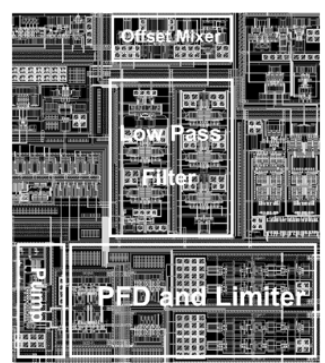

Fig. 9. Microphotograph of the Offset-PLL.

frequency, so the original down-converted signal, the VCO leakage, and the image at $f_{\mathrm{LO}}+f_{\mathrm{VCO}}$ can all be approximated as pure sinusoids at any instant in this phase-tracking process. Thus, the concept in Section II is still applicable. Suppose now that the instantaneous frequency of the modulated input is $f_{\text {ref }}+\Delta f$; then the $\mathrm{VCO}$ frequency is $f_{\mathrm{VCO}}+\Delta f$ and their relation can be written as

$$
\begin{aligned}
& \left(f_{\mathrm{ref}}+\Delta f\right)\left(\frac{1}{f_{\mathrm{ref}}+\Delta f}-N \frac{1}{f_{\mathrm{VCO}}+\Delta f}\right) \\
& =\left(f_{r_{\mathrm{vco}}}-(N-1) \Delta f\right) \frac{1}{f_{\mathrm{VCO}}+\Delta f}
\end{aligned}
$$

where $f_{\mathrm{VCO}}=N f_{\text {ref }}+f_{r_{\mathrm{vco}}}, 0 \leq f_{r_{\mathrm{vco}}}<f_{\text {ref }}$, and $0 \leq$ $f_{r_{\mathrm{vco}}} \mp(N-1) \Delta f<f_{\text {ref }} \pm \Delta f$.

Compared with (2), we can see that, when the instantaneous frequency of the modulated input varies from $f_{\text {ref }}-\Delta f$ to $f_{\text {ref }}+$ $\Delta f$, the residual frequency of the leaked VCO disturbance will vary correspondingly in the following frequency range:

$$
f_{r_{\mathrm{vco}}}+(N-1) \Delta f \geq f \geq f_{r_{\mathrm{vco}}}-(N-1) \Delta f .
$$

Similarly, since $f_{\mathrm{LO}}=f_{\mathrm{VCO}}+f_{\text {ref }}$ in this design, if $2 f_{r_{\mathrm{vco}}}>$ $f_{\text {ref }}$, the residual frequency of the image disturbance will vary in the frequency range

$$
f_{r_{\mathrm{vco}}}+(2 N+1) \Delta f \geq f \geq f_{r_{\mathrm{vco}}}-(2 N+1) \Delta f .
$$

Obviously, now that the residual frequencies are also modulated, the strength of spurs decreases. Indeed, compared to that in the case of applying a sinusoidal input, the strength will be decreased by more than $10 \mathrm{~dB}$ when the input is GMSK modulated in GSM applications. This is evident since, compared to the sinusoid-input case, there is about $10 \mathrm{~dB}$ down in the fundamental output power level in this GMSK-modulation case.

\section{OPLL OUTPUt SPUR MEASUREMENTS AND VALIDATION}

As explained previously, when sinusoidal IF is applied, the practical OPLL circuit presents output spurs at the following offsets:

$$
f_{\mathrm{spur}}= \pm\left(m f_{r_{\mathrm{vco}}}-n f_{\mathrm{ref}}\right)
$$

where $m=1$ or 2 and $n$ is any integer not very large.

In the following, experimental results are demonstrated to validate the proposed predictions. The measured OPLL circuit is fabricated in the TSMC $0.35-\mu \mathrm{m} \mathrm{SiGe} \mathrm{BiCMOS} \mathrm{technology}$ with the microphotograph shown in Fig. 9.

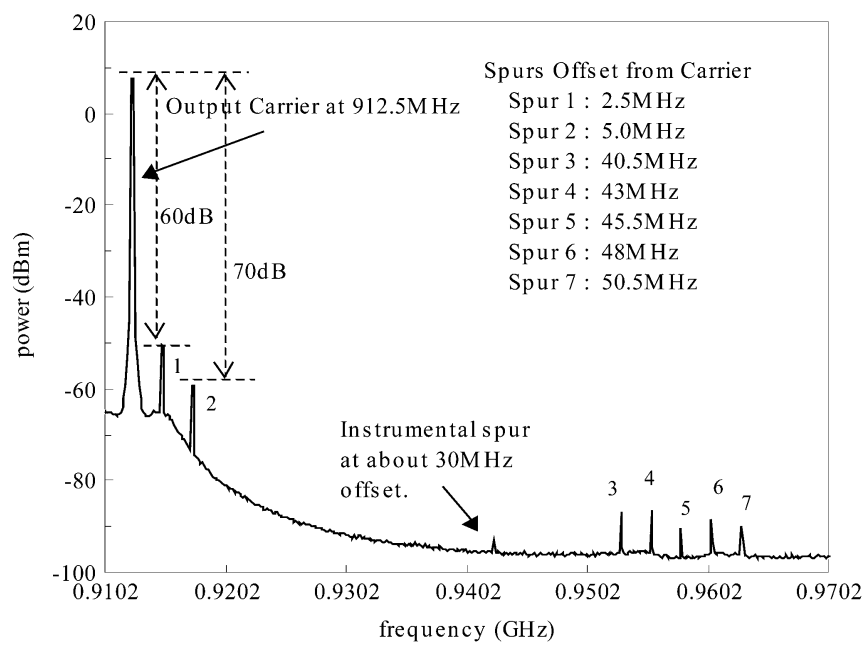

Fig. 10. Measured OPLL output spur spectrum with IF $=45.5 \mathrm{MHz}$ and $\mathrm{LO}=958 \mathrm{MHz}$

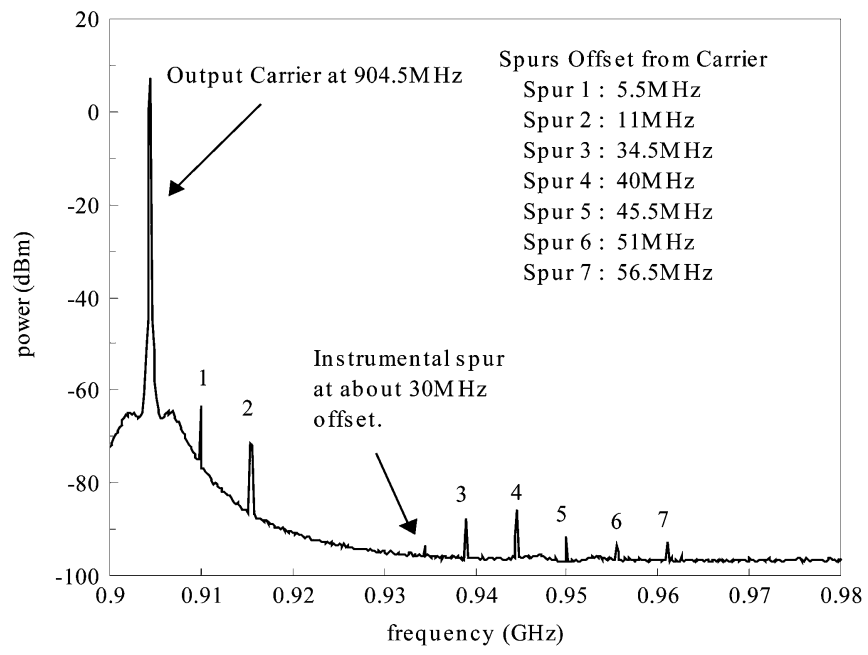

Fig. 11. Measured OPLL output spur spectrum with IF $=45.5 \mathrm{MHz}$ and $\mathrm{LO}=950 \mathrm{MHz}$.

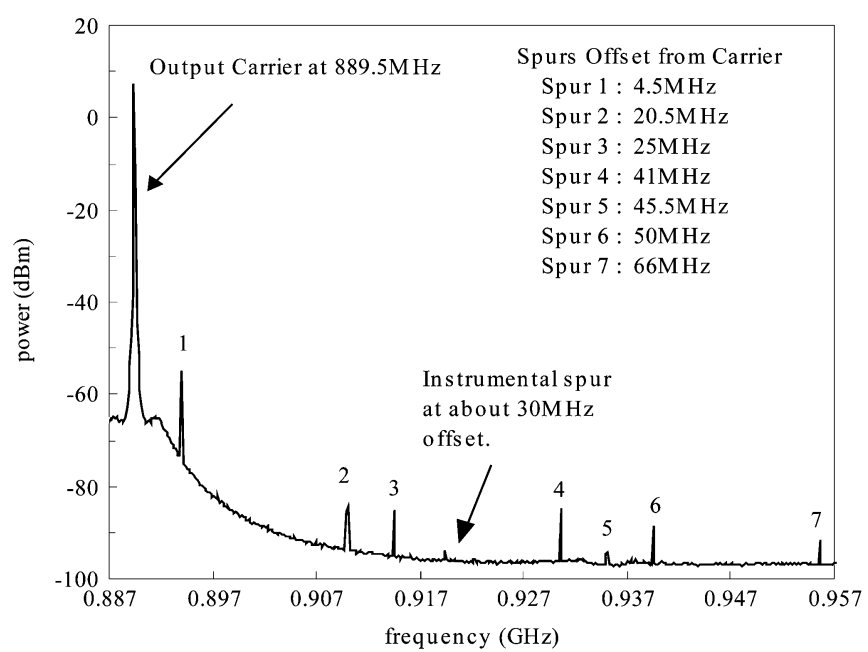

Fig. 12. Measured OPLL output spur spectrum with IF $=45.5 \mathrm{MHz}$ and $\mathrm{LO}=935 \mathrm{MHz}$.

In the measurements shown in Figs. 10-12, the reference signals are all set to $45.5 \mathrm{MHz}$ and the $\mathrm{LO}$ frequencies are 958 , 950, and $935 \mathrm{MHz}$, respectively. The corresponding RF VCO 
TABLE I

Spur Positions Shown in Figs. 10-12 AND the Predictions IN (18)

\begin{tabular}{|c|c|c|c|c|c|c|}
\hline \multicolumn{2}{|c|}{ Fig. 10} & \multicolumn{2}{|c|}{ Fig. 11} & \multicolumn{3}{|c|}{ Fig. 12} \\
\hline \multicolumn{2}{|c|}{$\begin{array}{l}\mathrm{LO}=958 \mathrm{MHz} \\
\mathrm{VCO}=912.5 \mathrm{MHz} \\
\mathrm{IF}=45.5 \mathrm{MHz}\end{array}$} & \multicolumn{2}{|c|}{$\begin{array}{l}\mathrm{LO}=950 \mathrm{MHz} \\
\mathrm{VCO}=904.5 \mathrm{MHz} \\
\mathrm{IF}=45.5 \mathrm{MHz}\end{array}$} & \multicolumn{3}{|c|}{$\begin{array}{l}\mathrm{LO}=935 \mathrm{MHz} \\
\mathrm{VCO}=889.5 \mathrm{MHz} \\
\mathrm{IF}=45.5 \mathrm{MHz}\end{array}$} \\
\hline$f_{r_{\mathrm{vco}}}=$ & $\mathrm{MHz}$ & $f_{r_{\mathrm{vco}}}=4$ & $\mathrm{MHz}$ & $f_{r_{\mathrm{vco}}}=2$ & $\mathrm{MHz}$ & \\
\hline Measured offset & Predicted in (18) & Measured offset & Predicted in (18) & Measured offset & Predict & in (18) \\
\hline $2.5 \mathrm{MHz}$ & $\mathrm{m}=1 \quad \mathrm{n}=0$ & $5.5 \mathrm{MHz}$ & $\mathrm{m}=1 \quad \mathrm{n}=1$ & $4.5 \mathrm{MHz}$ & $\mathrm{m}=2$ & $\mathrm{n}=1$ \\
\hline $5.0 \mathrm{MHz}$ & $\mathrm{m}=2 \quad \mathrm{n}=0$ & $11 \mathrm{MHz}$ & $\mathrm{m}=2 \quad \mathrm{n}=2$ & $20.5 \mathrm{MHz}$ & $\mathrm{m}=1$ & $\mathrm{n}=1$ \\
\hline $40.5 \mathrm{MHz}$ & $\mathrm{m}=2 \quad \mathrm{n}=1$ & $34.5 \mathrm{MHz}$ & $\mathrm{m}=2 \quad \mathrm{n}=1$ & $25 \mathrm{MHz}$ & $\mathrm{m}=1$ & $\mathrm{n}=0$ \\
\hline $43 \mathrm{MHz}$ & $\begin{array}{ll}m=1 & n=1\end{array}$ & $40 \mathrm{MHz}$ & $m=1 \quad n=0$ & $41 \mathrm{MHz}$ & $\mathrm{m}=2$ & $\mathrm{n}=2$ \\
\hline $45.5 \mathrm{MHz}$ & $\mathrm{m}=0 \quad \mathrm{n}= \pm 1$ & $45.5 \mathrm{MHz}$ & $\mathrm{m}=0 \quad \mathrm{n}= \pm 1$ & $45.5 \mathrm{MHz}$ & $\mathrm{m}=0$ & $\mathrm{n}= \pm 1$ \\
\hline $48 \mathrm{MHz}$ & $m=1 \quad n=-1$ & $51 \mathrm{MHz}$ & $\mathrm{m}=1 \quad \mathrm{n}=2$ & $50 \mathrm{MHz}$ & $\mathrm{m}=2$ & $\mathrm{n}=0$ \\
\hline $50.5 \mathrm{MHz}$ & $\mathrm{m}=2 \quad \mathrm{n}=-1$ & $56.5 \mathrm{MHz}$ & $\mathrm{m}=2 \quad \mathrm{n}=3$ & $66 \mathrm{MHz}$ & $\mathrm{m}=1$ & $\mathrm{n}=2$ \\
\hline
\end{tabular}

signals are then at $912.5,904$, and $889.5 \mathrm{MHz}$ with their respective residual frequencies at 2.5, 40, and $25 \mathrm{MHz}$. As listed in Table I, all of the spurs marked in these figures can be exactly predicted by (18).

Note that, in these measurements, the spurs at $45.5 \mathrm{MHz}$ offset are also observed. It is mainly due to circuit mismatches as explained previously and can be included in (18) by setting $m=0$ and $n=-1$. Note also that, in the experiments, the maximum output power of VCO is in fact about $10 \mathrm{dBm}$. To show up any tiny spurs on the monitor, the maximum power level on the original display is set to $0 \mathrm{dBm}$. Due to the signal-processing problem in the instrument, this clamp also generates a very little spur at about $30-\mathrm{MHz}$ offset in the measurements.

To clearly observe the scattering phenomenon of a single spur due to the input modulation, the unbalanced VCO output signal is fed to the offset mixer in experiments. This results in severe disturbance problems in OPLL. Fig. 13 shows the output spectrums in this setting before and after input modulation. Note that, in this situation, the VCO leakage is much larger than the LO leakage, thus the disturbances in OPLL are mainly from the leaked $\mathrm{VCO}$ signal and the image signal at $f_{\mathrm{LO}}+f_{\mathrm{VCO}}$. In Fig. 13(a), LO is set to $958 \mathrm{MHz}$ and the input IF before modulation is at $45.5 \mathrm{MHz}$. The nearest spurs at $\pm 2.5 \mathrm{MHz}$ apart from the carrier are apparently due to the VCO leakage since $f_{\mathrm{VCO}}=20 \times 45.5 \mathrm{MHz}+2.5 \mathrm{MHz}$. In GSM applications, the maximum frequency deviation $\Delta f$ almost equals to $0.068 \mathrm{MHz}$ [13]. Thus, according to (16), with $\mathrm{N}=20$ and $\Delta f=0.068 \mathrm{MHz}$, the spurs at $\pm 2.5 \mathrm{MHz}$ will, roughly speaking, be scattered over $\pm 1.292 \mathrm{MHz}$ around their original positions by the input modulation. As illustrated, the distribution of the spectrum pollution on the left-hand side ends at about a $-1.2-\mathrm{MHz}$ offset from the carrier. Apparently, the difference between this position to the nearest spur on the left-hand side of the carrier is $1.3 \mathrm{MHz}$. This value is quite close to the predicted $1.292 \mathrm{MHz}$. Similarly, in Fig. 13(b), the LO is set to $934.9 \mathrm{MHz}$ and the IF before modulation is again at $45.5 \mathrm{MHz}$. Obviously, the nearest spurs at $\pm 4.3 \mathrm{MHz}$ apart from the carrier are due to the image disturbance since $f_{\mathrm{VCO}}=19 \times 45.5 \mathrm{MHz}+24.9 \mathrm{MHz}$ and $2 \times 24.9>45.5$. According to (17), the modulation will roughly scatter the spurs over $\pm 2.652 \mathrm{MHz}$ around their original positions. The observed difference between the position of the nearest spur on the left-hand side of the carrier and the frequency position where the distribution of the spectrum pollution ends on the left-hand side of the carrier is $2.7 \mathrm{MHz}$, again following the predicted $2.652 \mathrm{MHz}$ quite well.

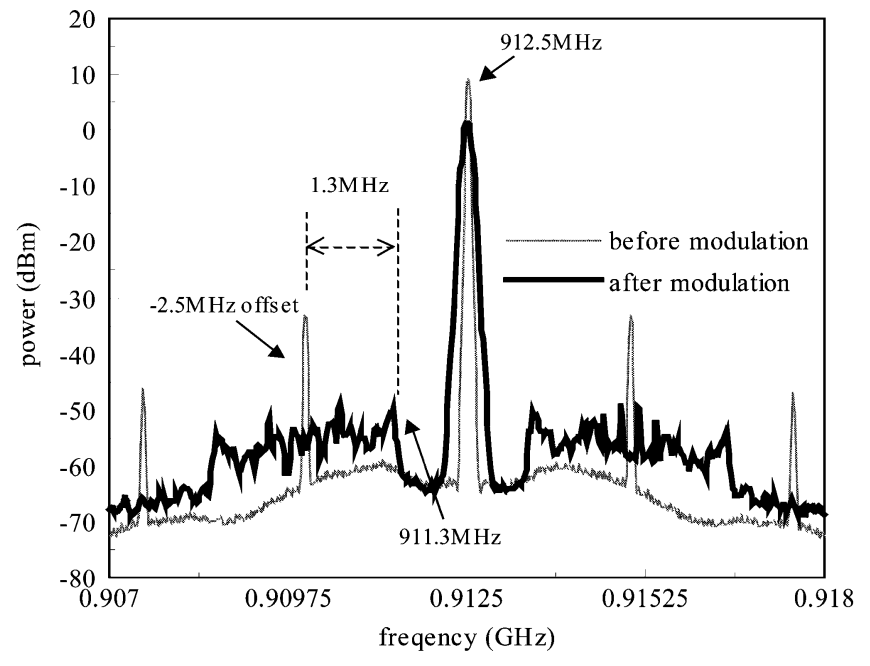

(a)

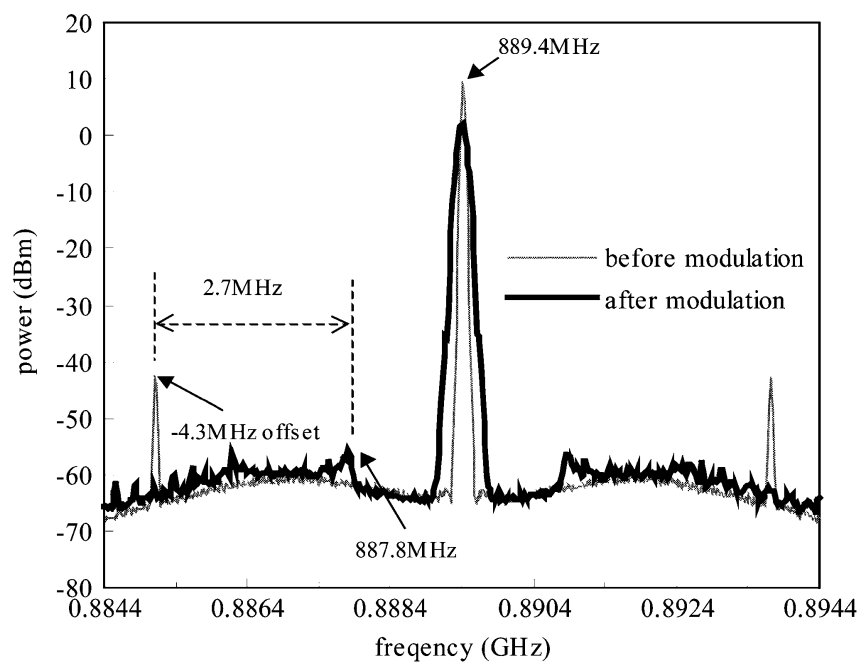

(b)

Fig. 13. Comparison of the measured OPLL spur spectrums before and after GMSK modulation at the input. (a) With IF $=45.5 \mathrm{MHz}$ and $\mathrm{LO}=958 \mathrm{MHz}$. (b) With IF $=45.5 \mathrm{MHz}$ and $\mathrm{LO}=934.9 \mathrm{MHz}$.

To evaluate the amplitude of spurs from the derived formula, we perturb the sinusoidal IF by an external disturbance. Note that the spurs appearing at the output are generated in the same mechanism as proposed in Section II, because now PFD also senses a periodic phase-deviation series. As shown in Fig. 14, 


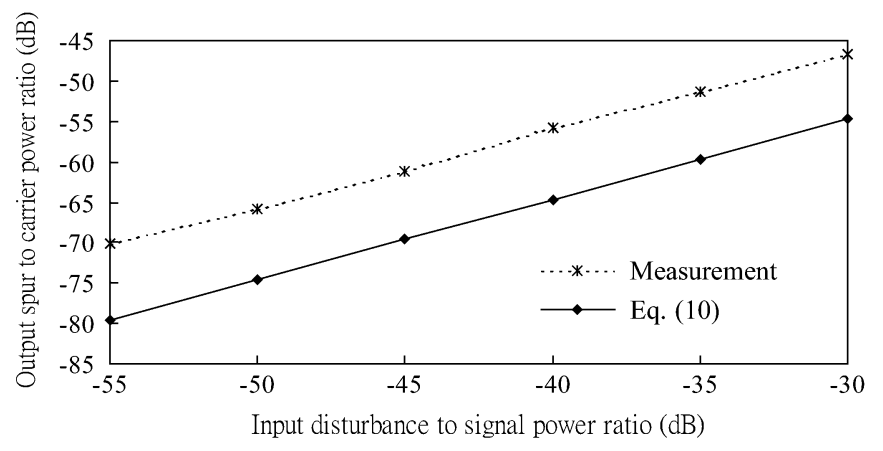

Fig. 14. Comparison between the measurement and the evaluation in (10) with $\mathrm{I}^{\mathrm{cP}}=2 \mathrm{~mA},\left|G_{\mathrm{lf}}(5 \mathrm{MHz})\right|=78 \Omega, K_{\mathrm{vco}}=2 \pi \times 23.5 \mathrm{MHz} / \mathrm{V}, \mathrm{IF}=$ $45.5 \mathrm{MHz}, f_{\text {disturb }}=915 \mathrm{MHz}$, and $f=5 \mathrm{MHz}$.

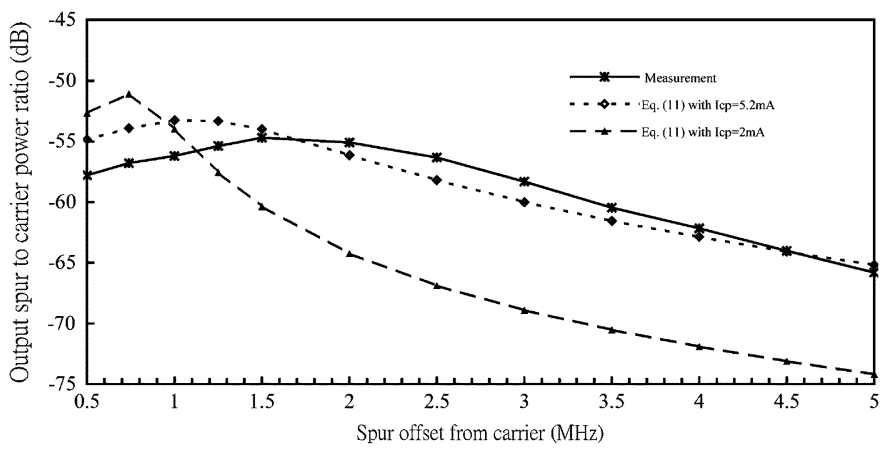

Fig. 15. In-band behavior of spurs obtained from measurement and (11) with $\mathrm{I}^{\mathrm{cp}}=2 \mathrm{~mA}$ and $5.2 \mathrm{~mA}$. Disturbance frequency sweeps from 910.5 to $915 \mathrm{MHz}$ and IF $=45.5 \mathrm{MHz}$. The power ratio of disturbance to reference is set to $-50 \mathrm{~dB}$.

the measured out-of-band spurs are about $8.3 \mathrm{~dB}$ larger than that expected. The discrepancy is due to the long turning-off time in the charging and sinking current pulses that makes the equivalent strength of each pulse about 2.6 times its original setting. The slower turning-off speed in each current pulse results from the inefficient discharge path in the pump driver. The increasing in the strength of current pulses also extends somewhat the loop bandwidth. The phenomenon is shown in Fig. 15, where the closed-loop behavior of spurs is measured and compared with the result in (11) with different $I_{\mathrm{cp}}$ 's.

The strength of disturbances delivered to the limiter can now be estimated from the measured Spurs 1 and 2 in Fig. 10 and (11) by using equivalent $I_{\mathrm{cp}}=5.2 \mathrm{~mA}$. After some algebraic operation, the power ratio of VCO leakage and image to the feedback signal at the limiter are found to be about -51.8 and $-55.85 \mathrm{~dB}$. This indicates the RF-band suppression by the lowpass filter is still not sufficient in this design. However, since the circuits are integrated in a silicon substrate, there are also many paths for the high-frequency disturbances to leak to the limiter and the PFD, and thus the layout also needs much attention.

\section{CONCLUSION}

In this paper, we present a new mechanism, by which valuable formulas are derived to predict the frequency positions of output spurs exactly and systematically. Some inherent characteristics are identified to be the foundation of the intermodulation nature of the output spur spectrum and methods are proposed to suppress the spurs from the viewpoint of circuit design and system-level considerations. Finally, the experimental results with both sinusoidal and modulated IF are demonstrated to validate the derived formulas with excellent agreement.

\section{APPENDIX A}

Equation (5) can be expanded at $d_{k}=0$ using Taylor's formula and approximated as

$$
\begin{aligned}
& 2\left(\pi f_{\text {dis }}\right)^{2} \sin \left(2 \pi f_{r} t+\phi_{\text {dis }}\right) d_{k}^{2} \\
& \quad+2 \pi\left[\frac{A}{B} f_{\text {ref }}+f_{\text {dis }} \cos \left(2 \pi f_{r} t+\phi_{\text {dis }}\right)\right] d_{k} \\
& \quad-\sin \left(2 \pi f_{r} t+\phi_{\text {dis }}\right)=0 .
\end{aligned}
$$

The solution $d_{k}$ in (19) can be approximated as

$$
d_{k} \cong \frac{\sin \theta}{c+b \cos \theta}-\frac{\frac{b^{2}}{2} \sin ^{3} \theta}{[c+b \cos \theta]^{3}}+\frac{\frac{b^{4}}{2} \sin ^{5} \theta}{[c+b \cos \theta]^{5}}
$$

where $a=2 \pi f_{r}, \theta=a t+\phi_{\text {dis }}, \mathrm{b}=2 \pi f_{\text {dis }}$, and $c=2 \pi f_{\text {ref }} A / B$.

Note that

$\sin \theta$

$\overline{c+b \cos \theta}$

$=\frac{\sin \theta}{c}\left[1-\frac{b}{c} \cos \theta+\left(\frac{b}{c} \cos \theta\right)^{2}-\left(\frac{b}{c} \cos \theta\right)^{3}+\cdots\right]$

$\cong \frac{1}{c} \sin \theta-\frac{1}{c} \frac{b}{c} \frac{1}{2} \sin 2 \theta+\frac{1}{c}\left(\frac{b}{c}\right)^{2} \frac{1}{4}(\sin 3 \theta+\sin \theta)$

$-\frac{1}{c}\left(\frac{b}{c}\right)^{3} \frac{1}{8}(\sin 4 \theta+2 \sin 2 \theta)$

$\frac{\frac{b^{2}}{2} \sin ^{3} \theta}{[c+b \cos \theta]^{3}}$

$=\frac{1}{2 c}\left(\frac{b}{c}\right)^{2}\left(\frac{3}{4} \sin \theta-\frac{1}{4} \sin 3 \theta\right)$

$\times\left[1-3 \frac{b}{c} \cos \theta+6\left(\frac{b}{c} \cos \theta\right)^{2}\right.$

$\left.-10\left(\frac{b}{c} \cos \theta\right)^{3}+15\left(\frac{b}{c} \cos \theta\right)^{4}+\cdots\right]$

$\cong \frac{1}{2 c}\left(\frac{b}{c}\right)^{2}\left\{\frac{3}{4}\left[\sin \theta-\frac{b}{c} \frac{3}{2} \sin 2 \theta+\left(\frac{b}{c}\right)^{2} \frac{3}{2}(\sin 3 \theta+\sin \theta)\right.\right.$

$-\frac{5}{4}\left(\frac{b}{c}\right)^{3}(\sin 4 \theta+2 \sin 2 \theta)$

$\left.+\frac{15}{16}\left(\frac{b}{c}\right)^{4}(\sin 5 \theta+3 \sin 3 \theta+2 \sin \theta)\right]$

$-\frac{1}{4}\left[\sin 3 \theta-\frac{b}{c} \frac{3}{2}(\sin 4 \theta+\sin 2 \theta)\right.$

$+\left(\frac{b}{c}\right)^{2} \frac{3}{2}(\sin 5 \theta+2 \sin 3 \theta+\sin \theta)$

$-\frac{5}{4}\left(\frac{b}{c}\right)^{3}(\sin 6 \theta+3 \sin 4 \theta+3 \sin 2 \theta)$

$+\frac{15}{16}\left(\frac{b}{c}\right)^{4}(\sin 7 \theta+4 \sin 5 \theta+6 \sin 3 \theta$

$+3 \sin \theta)]\}$ 


$$
\begin{aligned}
& \frac{\frac{b^{4}}{2} \sin ^{5} \theta}{[c+b \cos \theta]^{5}} \\
& =\frac{1}{2 c}\left(\frac{b}{c}\right)^{4}\left(\frac{11}{16} \sin \theta-\frac{5}{16} \sin 3 \theta+\frac{1}{8} \sin 5 \theta\right) \\
& \times\left[1-5 \frac{b}{c} \cos \theta+15\left(\frac{b}{c} \cos \theta\right)^{2}\right. \\
& \left.-35\left(\frac{b}{c} \cos \theta\right)^{3}+70\left(\frac{b}{c} \cos \theta\right)^{4}+\cdots\right] \\
& \cong \frac{1}{2 c}\left(\frac{b}{c}\right)^{4}\left\{\frac { 1 1 } { 1 6 } \left[\sin \theta-\frac{b}{c} \frac{5}{2} \sin 2 \theta\right.\right. \\
& +\left(\frac{b}{c}\right)^{2} \frac{15}{4}(\sin 3 \theta+\sin \theta)-\frac{35}{8}\left(\frac{b}{c}\right)^{3} \\
& \times(\sin 4 \theta+2 \sin 2 \theta) \\
& \left.+\frac{70}{16}\left(\frac{b}{c}\right)^{4}(\sin 5 \theta+3 \sin 3 \theta+2 \sin \theta)\right] \\
& -\frac{5}{16}\left[\sin 3 \theta-\frac{b}{c} \frac{5}{2}(\sin 4 \theta+\sin 2 \theta)\right. \\
& +\left(\frac{b}{c}\right)^{2} \frac{15}{4}(\sin 5 \theta+2 \sin 3 \theta+\sin \theta) \\
& -\frac{35}{8}\left(\frac{b}{c}\right)^{3}(\sin 6 \theta+3 \sin 4 \theta+3 \sin 2 \theta) \\
& \left.+\frac{70}{16}\left(\frac{b}{c}\right)^{4}(\sin 7 \theta+4 \sin 5 \theta+6 \sin 3 \theta+3 \sin \theta)\right] \\
& +\frac{1}{8}\left[\sin 5 \theta-\frac{b}{c} \frac{5}{2}(\sin 6 \theta+\sin 4 \theta)\right. \\
& +\left(\frac{b}{c}\right)^{2} \frac{15}{4}(\sin 7 \theta+2 \sin 5 \theta+\sin 3 \theta) \\
& -\frac{35}{8}\left(\frac{b}{c}\right)^{3}(\sin 8 \theta+3 \sin 6 \theta+3 \sin 4 \theta) \\
& \left.\left.+\frac{70}{16}\left(\frac{b}{c}\right)^{4}(\sin 9 \theta+4 \sin 7 \theta+6 \sin 5 \theta+3 \sin 3 \theta)\right]\right\} \text {. }
\end{aligned}
$$

When (21), $-1 \times(22)$, and (23) are summed together, (6) is obtained.

\section{APPENDIX B}

The Fourier series of total pulse sequence $Q_{s}(t)$ as depicted in Fig. 4 can be represented as

$$
\begin{aligned}
Q_{s}(t) & =\sum_{n=-\infty}^{\infty} c_{n} e^{j n \omega_{o} t}, \quad \omega_{\mathrm{o}}=\frac{2 \pi}{T} \\
c_{n} & =\sum_{k=0}^{\mathrm{K}_{\max }-1} I_{\mathrm{cp}} d_{k} \frac{\sin \left(n \pi d_{k} / T\right)}{n \pi d_{k}} e^{-j n \omega_{o}\left(T_{D_{k}}+\frac{\left|d_{k}\right|}{2}\right)}
\end{aligned}
$$

where $T=\mathrm{K}_{\max } T_{\text {ref }}, T_{D_{k}}=k T_{\text {ref }}-\left|d_{k}\right|$ for negative pulses, and $T_{D_{k}}=k T_{\text {ref }}$ for positive pulses.

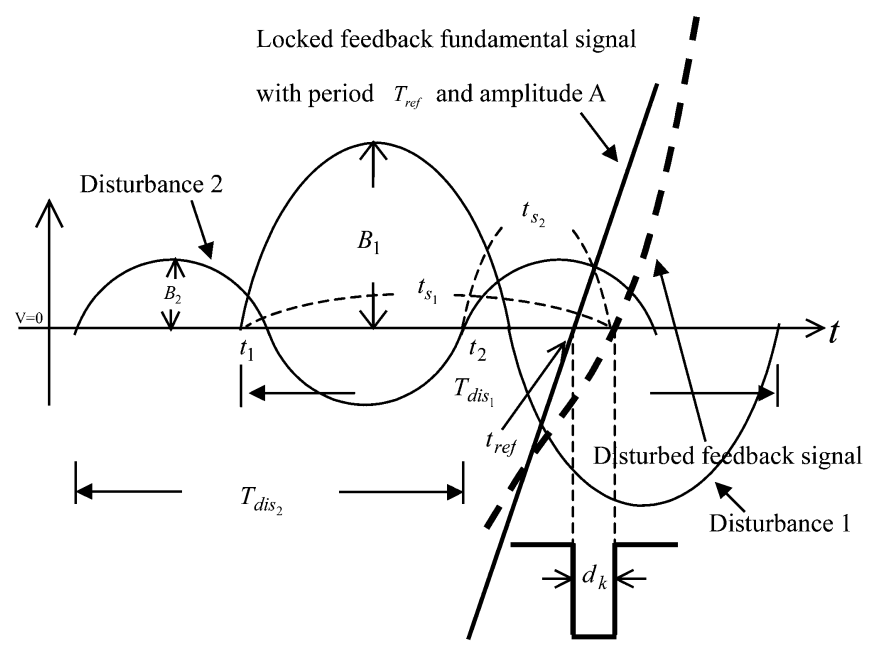

Fig. 16. Situation with two disturbances coexisting.

Since $\left|d_{k}\right|<<T_{D_{k}}<T$, the coefficient $c_{n}$ of $Q_{s}(t)$ in the low-frequency band can be approximated as

$$
\begin{aligned}
\mathrm{c}_{n} & \cong \sum_{k=0}^{\mathrm{K}_{\max }-1} \frac{I_{\mathrm{cp}} d_{k}}{T} e^{-j n \omega_{o} k T_{\text {ref }}} \\
& =\sum_{k=0}^{\mathrm{K}_{\max }-1} \frac{I_{\mathrm{cp}} d\left(k T_{\mathrm{ref}}\right)}{T} e^{-j n \omega_{o} k T_{\text {ref }}}
\end{aligned}
$$

where integers $n$ and $L$ satisfy $\left|n d_{k}\right|<\left|L d_{k}\right|<<T$.

The representation at the right-hand side of (25) is just the $n$th Fourier series coefficient of the function $I_{\mathrm{cp}} d(t)$ sampled at a rate of $f_{\text {ref }}$. The corresponding low-frequency signal $Q_{\text {low }}(t)$ is

$$
\begin{aligned}
Q_{\text {low }}(t)=\sum_{n=-L}^{L}\left(\frac{1}{T} \int_{0}^{T} \sum_{m=0}^{\mathrm{M}_{\max }-1} I_{\mathrm{cp}} d(\tau) \delta\right. \\
\\
\left.\quad \times\left(\tau-m T_{\mathrm{ref}}\right) e^{-j n \omega_{\mathrm{o}} \tau} \mathrm{d} \tau\right) e^{j n \omega_{o} t} .
\end{aligned}
$$

The Fourier transform of $Q_{\text {low }}(t)$ therefore is

$$
\begin{aligned}
F\left\{Q_{\text {low }}(t)\right\}= & I_{\mathrm{cp}} f_{\text {ref }} \sum_{n=-L_{2}}^{L_{2}} \mathbf{d}\left(2 \pi f+2 \pi n f_{\text {ref }}\right) \\
\cong & \frac{I_{\mathrm{cp}} B}{2 \pi A} \sum_{n=-L_{2}}^{L_{2}} \sum_{m=1}^{L_{1}} g_{m}\left(\frac{B}{A} \frac{f_{\text {dis }}}{f_{\text {ref }}}\right) \\
& \times\left[-j \pi \delta\left(f-m f_{r}+n f_{\text {ref }}\right) e^{j m \phi_{\text {dis }}}\right. \\
& \left.+j \pi \delta\left(f+m f_{r}+n f_{\text {ref }}\right) e^{-j m \phi_{\text {dis }}}\right]
\end{aligned}
$$

where $\mathbf{d}(2 \pi f)$ is the Fourier transform of $d(t)$. Thus, $Q_{\text {low }}(t)$ can be approximated as in (8).

\section{APPENDIX C}

For clarity, we assume that there are now two disturbances presenting as shown in Fig. 16. Then, the zero-crossing points of the disturbed feedback signal satisfies the following equation:

$A \sin \left(2 \pi \frac{d_{k}}{T_{\text {ref }}}\right)-B_{1} \sin \left(2 \pi \frac{t_{s_{1}}}{T_{\text {dis }_{1}}}\right)-B_{2} \sin \left(2 \pi \frac{t_{s_{2}}}{T_{\text {dis }_{2}}}\right)=0$ 


$$
\begin{aligned}
& d_{k} \cong \frac{\left(B_{1} \sin \theta_{1}+B_{2} \sin \theta_{2}\right)}{c+B_{1} b_{1} \cos \theta_{1}+B_{2} b_{2} \cos \theta_{2}}-\frac{\left(B_{1} \frac{b_{1}}{2}{ }^{2} \sin \theta_{1}+B_{2}{\frac{b_{2}}{2}}^{2} \sin \theta_{2}\right)\left(B_{1} \sin \theta_{1}+B_{2} \sin \theta_{2}\right)^{2}}{\left(c+B_{1} b_{1} \cos \theta_{1}+B_{2} b_{2} \cos \theta_{2}\right)^{2}} \\
& \frac{2\left(B_{1}{\frac{b_{1}}{2}}^{2} \sin \theta_{1}+B_{2}{\frac{b_{2}}{2}}^{2} \sin \theta_{2}\right)^{2}\left(B_{1} \sin \theta_{1}+B_{2} \sin \theta_{2}\right)^{3}}{\left(c+B_{1} b_{1} \cos \theta_{1}+B_{2} b_{2} \cos \theta_{2}\right)^{2}}
\end{aligned}
$$

where $B_{i}$ is the amplitude of the disturbance $i$, and $t_{s_{i}}=t_{\text {ref }}-$ $t_{i}-d_{k}=k T_{\mathrm{ref}} f_{r_{i}} T_{\mathrm{dis}_{i}}+t_{\phi_{i}}-d_{k}$.

Note that now the zero-cross point of the disturbed feedback signal may not be located in the $N_{i}+1$ cycle of the disturbance $i$. Following the same procedures in Appendix A, (28) can be approximated as

$$
\begin{array}{r}
\left(B_{1} \frac{b_{1}^{2}}{2} \sin \theta_{1}+B_{2}{\frac{b_{2}}{2}}^{2} \sin \theta_{2}\right) d_{k}^{2}+\left(c+B_{1} b_{1} \cos \theta_{1}+B_{2} b_{2} \cos \theta_{2}\right) d_{k} \\
-\left(B_{1} \sin \theta_{1}+B_{2} \sin \theta_{2}\right)=0
\end{array}
$$

where $b_{i}=2 \pi f_{\mathrm{dis}_{i}}, \theta_{i}=2 \pi f_{r_{i}} t+\phi_{\operatorname{dis}_{i}}, c=2 \pi f_{\text {ref }} A, t=$ $k T_{\text {ref }}$, and $k \in\left\{0,1,2, \ldots, \mathrm{K}_{\max }-1\right\}$.

Note that $\mathrm{K}_{\max }$ is the least common multiple of $p_{1}$ and $p_{2}$ in which $f_{\text {ref }} / f_{r_{1}}=p_{1} / q_{1}$ and $f_{\text {ref }} / f_{r_{2}}=p_{2} / q_{2}$. Thus, we have (30), shown at the top of this page.

Following the procedures similar to (21)-(23), it is not hard to find that the solution $d_{k}$ can be represented as

$$
\begin{aligned}
d_{k} \cong & \frac{1}{2 \pi f_{\mathrm{ref}}} \frac{B_{i}}{A} \sum_{i=1}^{2} \sum_{m=1}^{\infty} g_{m}\left(\frac{B_{i} f_{\mathrm{dis}_{i}}}{A f_{\mathrm{ref}}}\right) \sin \\
& \times\left(m\left(2 \pi f_{r_{i}} t+\phi_{\mathrm{dis}_{i}}\right)\right) \\
& +\sum_{\substack{m=-\infty \\
m \neq 0}}^{\infty} \sum_{\substack{\ell=-\infty \\
\ell \neq 0}}^{\infty} g_{m \ell}\left(B_{1}, B_{2}, f_{\mathrm{dis}_{1}}, f_{\mathrm{dis}_{2}}, A, f_{\mathrm{ref}}\right) \sin \\
& \left.\times\left(2 \pi\left(m f_{r_{1}}+\ell f_{r_{2}}\right) t+\phi_{m \ell}\right)\right)
\end{aligned}
$$

where $g_{m}$ 's are what's listed in (6) and $g_{m \ell}$ 's represent the intermodulation coefficients which are quite small when evaluated at the operating variables $B_{i}, f_{\mathrm{dis}_{i}}, A$, and $f_{\text {ref }}$.

\section{APPENDIX D}

Referring to Fig. 1, let the reference at "a" be $A_{o} \sin \left(2 \pi f_{\text {ref }} t\right)$ and assume a locked state the low frequency signal at "c" is $C \sin \left(2 \pi f_{r} t+\phi\right)$, then the signal at "d" is $\left|G_{\mathrm{lf}}\right| C \sin \left(2 \pi f_{r} t+\phi+\phi_{\mathrm{lf}}\right)$ where $\left|G_{\mathrm{lf}}\right| \angle \phi_{\mathrm{lf}}$ is the gain of loop filter, and the signal at "e" is $A_{\mathrm{vco}} \cos \left[2 \pi f_{\mathrm{vco}} t+\right.$ $\left.\phi_{\mathrm{vco}}+K_{\mathrm{vco}} \int\left|G_{\mathrm{lf}}\right| C \sin \left(2 \pi f_{r} t+\phi+\phi_{\mathrm{If}}\right) d t\right]$, which equals $A_{\mathrm{vco}}\left[\cos \left(2 \pi f_{\mathrm{vco}} t+\phi_{\mathrm{vco}}\right) \pm\left(K_{\mathrm{vco}}\left|G_{\mathrm{lf}}\right| C\right) /\left(4 \pi f_{r}\right)\right.$ $\left.\cos \left(2 \pi\left(f_{\mathrm{vco}} \pm f_{r}\right) t+\phi_{\mathrm{vco}} \pm \phi \pm \phi_{\mathrm{lf}} \mp(\pi / 2)\right)\right]$. Assume that the LO signal is $\sin \left(2 \pi f_{\mathrm{LO}} t+\phi_{\mathrm{LO}}\right)$ and the conversion gain of the mixer is $G_{c}$, and then the total signal at "b" is the disturbance plus $A \sin \left(2 \pi f_{\text {ref }} t\right) \pm$ $A\left(K_{\mathrm{vco}}\left|G_{\mathrm{lf}}\right| C\right) /\left(4 \pi f_{r}\right) \sin \left(2 \pi\left(f_{\mathrm{ref}} \mp f_{r}\right) t \mp \phi \mp \phi_{\mathrm{lf}} \pm(\pi) /(2)\right)$, where $A=\left|G_{\mathrm{lp}}\right| G_{c} A_{\mathrm{vco}}, \phi_{\mathrm{LO}}-\phi_{\mathrm{vco}}=-\phi_{\mathrm{lp}}$, and the gain of the low-pass filter is assumed to be $\left|G_{\mathrm{lp}}\right| \angle \phi_{\mathrm{lp}}$ for signals at frequencies $f_{\text {ref }}$ and $f_{\text {ref }} \pm f_{r}$. According to (8) and the concept in Appendix $\mathrm{C}$, we find that, for the original disturbance $B \sin \left(2 \pi f_{\text {disturb }} t\right), C \sin \left(2 \pi f_{r} t+\phi\right)=$ $\left(I_{\mathrm{cp}}\right) /(2 \pi) g_{1}\left[(B / A) \sin \left(2 \pi f_{r} t\right)-\left(K_{\mathrm{vco}}\right) /\left(2 \pi f_{r}\right)\left|G_{\mathrm{lf}}\right| C\right.$ $\left.\sin \left(2 \pi f_{r} t+\phi+\phi_{\text {lf }}-(\pi / 2)\right)\right]$. Thus, the power ratio in (11) can be obtained. Note that the derivations above assume that the spurs at $\pm f_{r}$ are dominant and the feedback of other spurs is negligible.

\section{ACKNOWLEDGMENT}

C. F. Lee would like to thank T. Yang, J.-M. Hsu, S. Su, S.-Jean Liao, P. Su, and J.-L. Liu, ITRI, Hsinchu, Taiwan, for their kind assistance.

\section{REFERENCES}

[1] G. Irvine, S. Herzinger, R. Schmidt, D. Kubetzko, and J. Fenk, "An up-conversion loop transmitter IC for digital mobile telephones," in IEEE Int. Solid-State Circuits Conf. Dig. Tech. Papers, Feb. 1998, pp. 364-365.

[2] J. Dekosky, F. Martin, and J. Rollman, "Offset PLL analysis can cut spurious levels," Microw. RF, vol. 38, no. 11, pp. 107-112, Nov. 1999.

[3] M. A. Margarit and M. J. Deen, "A low power high spectral purity frequency translational loop for wireless application," in Proc. IEEE Custom Integrated Circuits Conf., May 2000, pp. 593-596.

[4] J. M. Hsu, "A 0.18- $\mu \mathrm{m}$ CMOS offset-PLL upconversion modulation loop IC for DCS1800 transmitter," IEEE J. Solid-State Circuits, vol. 38 , no. 4, pp. 603-613, Apr. 2003.

[5] T. Yamawaki, M. Kokubo, and H. Hagisawa, "A CMOS offset phase locked loop for a GSM transmitter," Analog Integrat. Circuits Signal Process., vol. 25, pp. 253-259, Dec. 2000.

[6] A. Hajimiri, "Noise in phase-locked loops for a GSM transmitter," in Proc. Southeast Symp. Mixed-Signal Design, Feb. 2001, pp. 1-6.

[7] B. Razavi, RF Microelectronics. Upper Saddle River, NJ: PrenticeHall, 1998

[8] R. Ahola, J. Routama, S. Lindfors, and K. Halonen, "A phase detector with no dead zone and a very wide output voltage range charge pump," in Proc. IEEE Int. Symp. Circuits Systems, vol. 1, Jun. 1998, pp. 155-158.

[9] G. Arfken and H. Weber, "Bessel functions," in Mathematical Methods for Physicists, 4th ed. New York: Academic, 1995, ch. 11, pp. $627-629$

[10] N. Morrison, Introduction to Fourier Analysis. New York: Wiley, 1994.

[11] RF Transceiver IC for GSM and PCN Dual Band Cellular Systems-HD155 121F Datasheet. San Jose, CA: Hitachi Semiconductor America Inc., 2000.

[12] ENFVZ4G59 Datasheet, 2002.

[13] Digital Cellular Telecommunications System (Phase 2+); Mobile Station (MS) Conformance Specification; Part 1: Conformance Specification (GSM 11.10-1 Version 6.0.0 Release 1997), 1997. Eur. Telecommun. Standards Inst.

[14] Digital Cellular Telecommunications System (Phase 2+); Radio Transmission and Reception (GSM 05.05 Version 5.7.1): Eur. Telecommun. Standards Inst., 1998. 


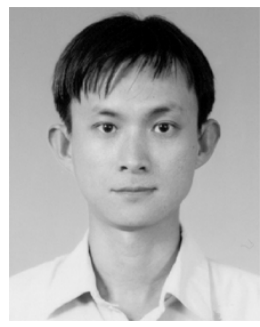

Ching-Feng Lee was born in Kaohsiung, Taiwan, R.O.C., in 1967. He received the B.S. degree in control engineering from the National Chiao-Tung University, Hsinchu, Taiwan, in 1989, and the M.S. degree from the Institute of Electrical Engineering, National Taiwan University, Taipei, Taiwan, in 1991. $\mathrm{He}$ is currently working toward the Ph.D. degree at the National Chiao-Tung University.

From 1991 to 1998, he was with the Computer and Communication Research Laboratory, ITRI, Hsinchu, Taiwan, where he was involved in power converter design, electrical analysis and design in backplanes and packages, and the discrete RF front-end circuit design. In 1998, he jointed ERSO, ITRI, where he was involved in the RF transceiver design. He holds a U.S. patent on the method of FM demodulation.

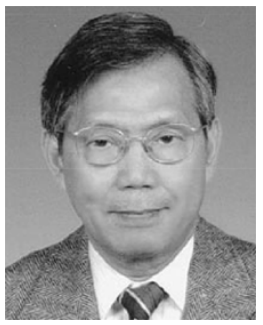

Song Tsuen Peng (M'74-SM'82-F'88) was born in Taiwan, R.O.C., on February 19, 1937. He received the B.S. degree in electrical engineering from the National Cheng Kung University, Tainan, Taiwan, in 1959, the M.S. degree in electronics from the National Chiao-Tung University, Hsinchu, Taiwan, in 1961, and the Ph.D. degree in electrophysics from the Polytechnic Institute of Brooklyn, Brooklyn, NY, in 1968.

Following 1968, he held various research positions with the Polytechnic Institute of Brooklyn. From 1983 to 1990, he was a Professor of electrical engineering and Director of the Electromagnetics Laboratory, New York Institute of Technology, Old Westbury. Since 1991, he has been a Professor of communication engineering with the National Chiao-Tung University. Since September 1998, he has been the Director of the Microelectronics and Information Systems Research Center, National Chaio-Tung University. He has been active in the field of general waveguiding structures and has published numerous papers on electromagnetics, optics, and acoustics. His current research interests include the guidance and scattering characteristics of periodic structures, antenna design, and electromagnetic compatibility.

Dr. Peng is a member of Sigma Xi. 